\title{
THE 488,006,860 SOURCES IN THE USNO-A1.0 CATALOG
}

\author{
B. CANZIAN \\ USRA/U. S. Naval Observatory, Flagstaff
}

\begin{abstract}
The USNO-A1.0 catalog was generated from the U. S. Naval Observatory's digitization of the Palomar Observatory Sky Survey I $O$ and $E$ survey plates for fields with central declination $\delta \geq-30^{\circ}$, and from the European Southern Observatory $R$ and Science Research Council $J$ survey plates for fields with central $\delta \leq-35^{\circ}$ using the Precision Measuring Machine (PMM) located at the Flagstaff Station. It lists positions ( $\alpha$ and $\delta$ in J2000) and brightnesses (red and blue magnitudes on the parent plate system) for all objects. A flag indicates if the entry also exists in GSC1.1.
\end{abstract}

\section{Object Distribution}

The PMM detected and measured objects at and beyond the nominal visual limiting magnitudes of $O=21, E=20, J=22$, and $F=21$. USNO-A1.0 was constructed from the correlation of the blue and red detection lists, and the only acceptance requirement was that there be matching detections with a 1 arcsec radius aperture. The object classification information was ignored: USNO-A1.0 is a catalog of astrometric standards.

The plot of the sky shown in Figure 1 was generated from the 488,006,860 sources contained in the USNO-A1.0 catalog. The sky is shown in Galactic coordinates (Galactic Center at center, north up, longitude increasing to the right) and the shading is proportional to the logarithm of the number of catalog sources per square degree. The increase in brightness in the vicinity of the south equatorial pole arises from the approximate factor of two increase in the number of PMM detections found on Southern survey plates as compared to Northern survey plates. The Large and Small Magellanic Clouds are obvious and dust in nearby clouds obscures parts of the Galactic plane. Dark spots mark the brightest stars in the sky, where detections are suppressed owing to flare on the plates. 


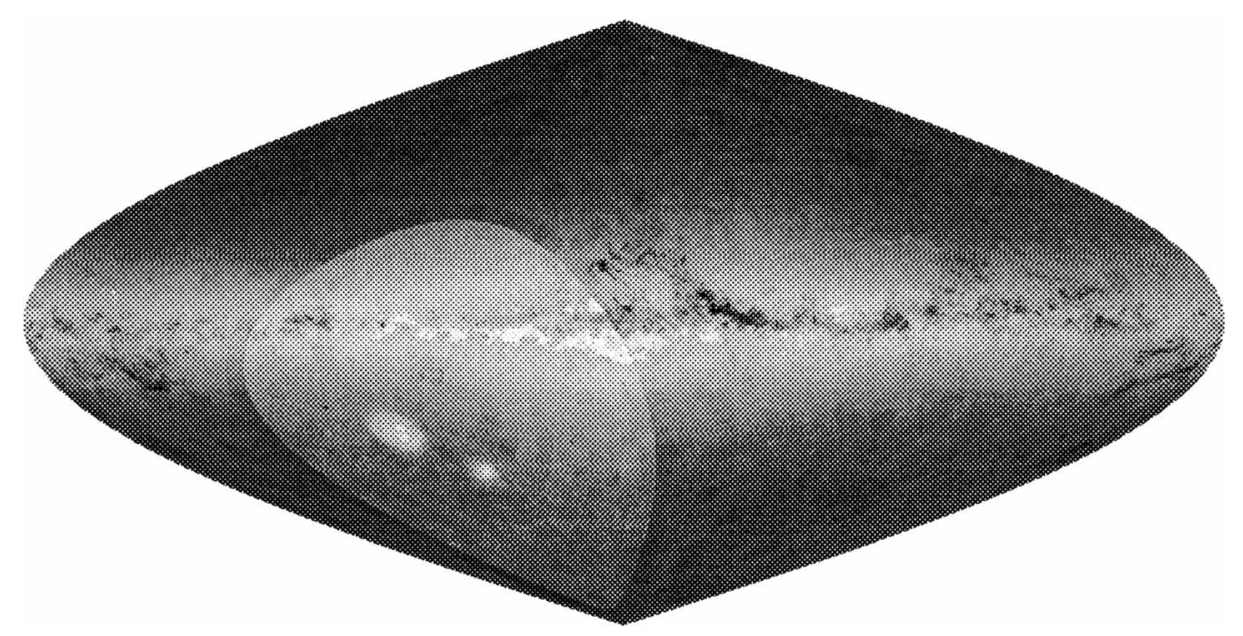

Figure 1. This is a representation of all the objects in the USNO-A1.0 catalog. The grayscale is coded to the logarithm of star density with black equal to 2.8 (about $700 \mathrm{deg}^{-2}$ ) and white equal to 5.1 (about $130,000 \mathrm{deg}^{-2}$ ).

\section{Calibration}

The USNO-A1.0 astrometry has been reduced with respect to GSC1.1, and so retains all the systematic errors of that catalog. Nonetheless, the USNO-A1.0 catalog is useful as a deep, all-sky reference catalog. The mean dispersion of the astrometric error in USNO-A1.0 is about 0.4 arcsec.

The bright photometry of USNO-A1.0 has been calibrated using the Tycho input catalog. The faint photometry has been calibrated using CCD photometry of 185 fields in common with the USNO parallax program. Systematic differences from plate to plate in the photometric slopes and zero points were removed by relaxing all plates to a common system using their overlap regions. The dispersion of the photometric error for faint $\left(13-21^{\text {st }}\right.$ magnitude) stars is about $0.3 \mathrm{mag}$.

\section{Acknowledgements}

The USNO PMM has been funded by the U. S. Navy and Air Force, and USNO-A1.0 incorporates intellectual property rights held by Palomar Observatory, California Institute of Technology, the National Geographic Society, European Southern Observatory, the Particle Physics and Astronomy Research Council, the Anglo Australian Observatory, and the Space Telescope Science Institute. 